\title{
Twelve DNA markers accurately assess risk of progression in adolescent idiopathic scoliosis \\ JT Braun
}

Address: Department of Orthopaedics and Rehabilitation, University of Vermont, Burlington, VT, USA

Email: JT Braun - john.braun@earthlink.net

from 4th International Conference on Conservative Management of Spinal Deformities

Boston, MA, USA. 13-16 May 2007

Published: 12 October 2007

Scoliosis 2007, 2(Suppl I):S4I doi:I0.II86/I748-7|6I-2-SI-S4I

This abstract is available from: http://www.scoliosisjournal.com/content/2/SI/S4 I

(c) 2007 Braun; licensee BioMed Central Ltd.

\section{Objective}

Optimization of AIS management necessitates early and accurate identification of progressive deformities. As standard radiographic evaluation relies on variables that change over time, these methods are often inaccurate in assessing risk of progression. Most data suggest that adolescent idiopathic scoliosis (AIS) has a polygenic inheritance. The exact gene(s) involved, however, have not been identified. Using our unique genealogy resources and a large patient population from across the United States, we hope to discover the molecular basis of AIS. As an interim result in our efforts to understand the genetic basis of AIS, we report on twelve DNA markers potentially useful in accurately predicting curve progression.

\section{Methods}

DNA samples from 675 skeletally mature AIS subjects were collected from spine centers across the U.S. Radiographic data at initial presentation during adolescence and at skeletal maturity allowed identification of surgical curves (progression beyond 40 degrees Cobb). Genotypes were obtained for twelve DNA markers identified as having diagnostic utility in AIS. Genotype weighting factors were established in an independent discovery sample set. Clinical risk scores were estimated using the subject's first radiographic evaluation for AIS. Using an additive model, summed risk scores were used to classify subjects as either LOW or HIGH risk for progression to a surgical curve.

\section{Results}

Of 675 subjects with AIS, 454 progressed to a surgical curve (>40 degrees), with 187 and 34, respectively, maintaining mild (<25 degrees) and moderate (25-40 degrees) curves. Using the additive model, 181/187 (97\%) of subjects with mild curves were correctly classified as LOW risk and $416 / 454(92 \%)$ as HIGH risk for progression to a surgical curve $(\mathrm{p}<0.00001)$.

\section{Conclusion}

The twelve DNA markers discovered in this study will greatly improve our ability to assess risk of progression. For HIGH risk patients this will likely result in earlier bracing or fusionless scoliosis surgery. For LOW risk patients this will allow elimination of years of unnecessary observation. When compared to standard radiographic methods, the twelve DNA markers identified in this study provide a superior assessment of risk of progression in AIS. Additional research will likely refine this marker panel and further improve our ability to accurately predict AIS progression. 